Euskal ikerketen aldizkaria | Revue d'études basques |

Revista de estudios vascos | Basque studies review

$20 \mid 2017$

Numéro $X X$

\title{
De l'usage des périphrases surcomposées dans les textes anciens
}

\section{Céline Mounole}

\section{(2) OpenEdition}

\section{Journals}

Édition électronique

URL : https://journals.openedition.org/lapurdum/3515

DOI : 10.4000/lapurdum.3515

ISSN : 1965-0655

Éditeur

IKER

Édition imprimée

Date de publication : 1 janvier 2017

Pagination : 133-147

ISBN : 978-2-95534-135-3

ISSN : $1273-3830$

Référence électronique

Céline Mounole, « De l'usage des périphrases surcomposées dans les textes anciens », Lapurdum [En ligne], 20 | 2017, mis en ligne le 01 janvier 2021, consulté le 03 septembre 2021. URL : http:// journals.openedition.org/lapurdum/3515; DOI : https://doi.org/10.4000/lapurdum.3515 


\section{De l'usage des périphrases}

surcomposées dans les textes anciens

Céline Mounole UPV/EHU

\section{Introduction ${ }^{1}$}

Les périphrases surcomposées se constituent à partir d'un participe lexical, d'un participe être ou avoir (plus rarement répété deux ou trois fois) et d'un auxiliaire. C'est de ce participe « extra » que leur vient l'appellation de surcomposées.

Elles sont rares d'un point de vue typologique (Schaden 2009). ${ }^{2}$ On les retrouve dans les langues romanes -français (Harris 1988, Ayres-Bennett \& Carruthers 1992), occitan (Ronjat [1930-41] 1980, Wheeler 1988, Birabent \& Salles-Loustau 1989), les variétés septentrionales de l'italien (Schaden 2009)-, rhéto-romanes (Schaden 2009), slaves -bulgare, serbo-croate, tchèque, slovaque, polonais, serbe et russe-, germaniques -yiddish, dialectes méridionaux de l'allemand-, mais aussi en albanais -dialectes Geg-, arménien et persan moderne (Estaji \& Bybenik 2007).

Selon Comrie (1985 : 76), bien souvent il s'avère très difficile de prédire le recours aux formes surcomposées dans les langues qui en possèdent. Cependant, comme montré par l'étude de Schaden (2009), plus les descriptions des données des différentes langues seront précises, plus nous aurons d'éléments pour envisager des généralisations à visée typologique.

1. Cette recherche est financée par les projets suivants : « Monumenta Linguae Vasconum (IV): textos arcaicos vascos y euskera antiguo» (Ministère de la Recherche espagnol, FFI2012- 37696) et «Historia de la lengua vasca y lingüística histórico-comparada» (HLMV-LHC) (Gouvernement basque, GIC. IT698-13).

2. «De par leur fréquence d'apparition peu élevée et la faible conscience des locuteurs du français à leur égard, les temps surcomposés peuvent passer pour des temps rares et exceptionnels. Une toute autre question cependant est de savoir si les temps de ce type apparaissent comme rares ou exceptionnels d'un point de vue typologique ou théorique. La réponse à cette question est oui. » (Schaden 2009 : 187) 
Le présent article ne prétend pas offrir une étude exhaustive des formes surcomposées employées en basque ancien; il se limite aux emplois aspectuo-temporels, c'est-à-dire que les emplois conditionnels et passifs des formes surcomposés ne sont pas abordés. Le corpus étudié est lui aussi limité : il inclut Leizarraga et Lazarraga au $16^{\text {ème }}$ siècle, Beriain au $17^{\text {ème }}$ siècle, Otxoa Arin, Etxeberri de Sare et Maizter au $18^{\text {ème }}$ siècle. Nous entendons ainsi, dans l'attente d'une étude plus approfondie, livrer une première description des formes surcomposées du basque ancien, de leur formation et des valeurs qui y sont attachées (\$2), pour ensuite ouvrir de nouvelles perspectives sur leurs origine et évolution diachronique (\$3).

\section{Formes et valeurs des periphrases surcomposées des textes anciens ${ }^{3}$}

$\mathrm{Au} 16^{\mathrm{ème}}$ siècle, les périphrases surcomposées aspectuo-temporelles ne se rencontrent que chez le labourdin Leizarraga et l'alavais Lazarraga. ${ }^{4}$ Cette première constatation est intéressante à plusieurs égards. Tout d'abord, le fait que les textes bas-navarrais (Etxepare) et souletins (Oihenart, Zalgize) n'apportent aucune attestation de ces formes, alors qu'on les retrouve dans ces variétés les siècles suivants, pose la question de la pertinence du témoignage de Leizarraga. Lafon (1943), dans sa thèse, émet des doutes quant à la vivacité de ces formes dans la langue parlée de l'époque. ${ }^{5}$ Le zèle des réformateurs à fournir les traductions les plus fidèles au texte source est bien connue, et cela pourrait pousser à croire que les périphrases surcomposées, si fréquentes dans le Nouveau Testament de Leizarraga, sont des calques du texte originel (nous reviendrons sur ce point ultérieurement, \$3). Ensuite, le biscayen n’a pas développé de périphrases surcomposées et le manuscrit de Lazarraga est donc le premier et le seul texte de l'aire occidentale à en rapporter l'usage. C'est, d'ailleurs, une preuve supplémentaire de la distance linguistique entre le biscayen et l'alavais (Mounole 2014).

Aux $17^{\text {ème }}$ et $18^{\text {ème }}$ siècles, les périphrases surcomposées se relèvent en guipuzcoan (Otxoa Arin, doctrine de Zegama, Kardaberatz), haut-navarrais (Beriain), labourdin (Etxeberri de Sare) et souletin (Maister). Autrement dit, elles révèlent la même distribution dialectale que de nos jours.

Comme en basque moderne, le participe « extra » est invariablement izan « être » (joan izan naiz 'j'ai eu été/ egin izan dut 'j'ai eu fait') dans les dialectes centraux (Otxoa Arin, doctrine de Zegama, Beriain, Etxeberri de Sare), tandis que dans les dialectes plus orientaux (Leizarraga

3. Pour les gloses des exemples, nombreux dans ce paragraphe et le suivant, dans le cas de traductions (Leizarraga, Maizter, Etxeberri de Sare, Garibai), nous avons employé le texte source et mis la glose entre doubles gillemets. Les exemples des autres textes, exception faite de Etxepare pour lequel nous avons utilié la traduction de Lafon, nous les avons traduits nous-mêmes et encadrés par des gillemets simples.

4. Lauteur alavais n'apporte qu'un seul exemple de ces formes dans tout le manuscrit: içan ninçan / exil-exilic coladu (B12: 47-48); nous y reviendrons plus bas (\$2.2).

5. «On peut d'ailleurs douter que, en basque, les formes surcomposées aient jamais été très vivantes dans la langue parlée. Ni Dechepare ni Oihenart ne les emploient dans leurs poésies; et l'on n'en trouve ni dans les proverbes de Garibay, ni dans ceux de d'Oihenart, ni dans les Refranes de $1596 »$ (Lafon 1943-II : 121). 
et Maister), izan « être » n'apparaît qu'avec les participes lexicaux intransitifs (joan izan naiz 'j'ai eu été'), ukan « avoir» étant d'usage avec les transitifs (egin ukan dut 'j'ai eu fait'). Les langues voisines du basque telles que le français (Ayres-Bennett \& Carruthers 1992, Harris 1982, 1988) et l'occitan (Ronjat 1930-41, Wheeler 1988), elles, n'utilisent que avoir comme auxiliaire « extra ». Le cas des dialectes orientaux semble donc relever, encore une fois, de leur tendance à préserver l'alignement ergatif (Aldai 2008, Creissels \& Mounole 2015). Quant au participe lexical, il est invariable.

Dans les textes les plus anciens, les différents dialectes possèdent deux types de périphrases surcomposées, celles à auxiliaire de présent et celles à auxiliaire de passé. Il convient de les distinguer car non seulement les valeurs qui y sont attachées sont différentes, mais en plus, elles semblent issues de processus de grammaticalisation différents.

\subsection{Surcomposées à auxiliaire de présent}

Les périphrases surcomposées à auxiliaire de présent expriment :

(i) le parfait ${ }^{6}$ (Leizarraga, Etxeberri de Sare, Otxoa Arin, Maizter) (2-3). Il est intéressant de constater que dans le texte de Leizarraga, une grande partie de ces surcomposées à valeur de parfait apparaissent dans les déclarations des prophètes (4). ${ }^{\top}$

(ii) le parfait d'expérience ${ }^{8}$ (Leizarraga) (1).

(iii) le « parfait lointain $»^{9}$ (ou « superparfait $»^{10}$ ), c'est-à-dire l'état résultant d'un événement situé dans un passé lointain mais qui conserve des incidences au moment de l'énonciation (Leizarraga, Otxoa Arin, Etxeberri de Sare) (5-8). On

6. Nous entendons par «parfait» une situation produite dans le passé, mais dont les conséquences sont toujours pertinentes au moment de l'énonciation.

7. Néanmoins, la forme de parfait composée simple est également d'usage : Orduan compli cedin Hieremias prophetáz erran içan cena, cioela, eta hartu ukan dituzté hoguey eta hamar diruäc, estimatu içan denaren precioa, cein estimatu içan baita Israeleco haourréz : Eta eman dituzte hec tupinaguile baten landaren erosteco, Iaunac niri ordenatu cerautan beçala (Leiz. Mt. XXVII, 9-10).

8. "The experiential perfect indicates that a given situation has held at least once during some time in the past leading up to the present $»$ (Comrie 1976:58).

9. Terminologie déjà employée par Aldai (2002) (« remote perfect »).

10. Schaden (2009), en reprenant la terminologie de Régnier, le dénomme «superparfait ». Contrairement à nous, il ne parle pas explicitement de l'ancienneté de l'événement impliqué : «Ce que tous les cas de surcomposés de type superparfait ont en commun, à travers ces différentes langues, est qu'ils expriment toujours un état résultant qui ne tient plus, mais pour lequel on envisage des conséquences au moment de l'énonciation » (Schaden 2009 : 214). 
retrouve cet usage en occitan (Wheeler 1988). ${ }^{11}$

(iv) une itération, ou plus précisément un événement qui s'est fréquemment produit dans le passé et jusqu'au moment de l'énonciation (sorte de "parfait itératif »), ce qui explique le recours à une forme habituellement employée pour rendre le parfait (Leizarraga, Etxeberri de Sare, Maister) (9-12). ${ }^{12}$

(v) le passé narratif, ${ }^{13}$ au même titre que les périphrases [radical verbal + ${ }^{*}$ edin « devenir », *ezan], [participe passé + egin « faire »], ou [participe passé + izan « être » / *edun « avoir » de passé] (cf. Mounole 2011). ${ }^{14}$ D’ailleurs, la surcomposée ainsi employée est souvent entourée d'autres formes de passé narratif (Leizarraga) (13).

(vi) le passé lointain (Etxeberri de Sare, Maister) (14-15). On retrouve cet usage en gascon (Birabent \& Salles-Loustau 1989). ${ }^{15}$

(1) Ençun ukan duçue (Leiz. Mt, V, 27) « vous avez ouï dire »

(2) Eta hori icussiric discipuluéc mirests ceçaten, cioitela, nolatan bertan eyhartu içan da ficotzea? (Leiz. Mt. XXI, 20) «Les disciples, ayans veu cela, s'esmerueillerent, disans, comment est le figuier incontinent deuenu sec?»

(3) nola istorioac eta autoren isquiribuac baitira landa eder eta çabalac, ceinetan

11. «Firstly, related to the speaker's present, the sobrecompausat expresses present relevance of an event remote in time, in contrast to the present perfect, which expresses present relevance of a recently past event / state ; for example, ai agut vist 'Le dernier Tango à Paris' 'I have seen « Last Tango in Paris » (i.e.already / once upon a time / several times in the past'); vs ai vist 'Le dernier Tango à Paris' "I have seen 'Last Tango in Paris' » (i.e. recently / this week) ; i siatz aguda estada en Arle, vos? « Have you even beeen to Arles? » (Wheeler 1988 : 265)

12. Dans les variétés occidentales du basque moderne, la périphrase surcomposée à auxiliaire de présent exprime une habitude: Berrogei urtez holaxet izkiriatu ukan ditu bere berriak (Euskaltzaindia 1987: 464, Oyharçabal 2003 : 258). Bien que nous ne trouvions pas cette valeur dans les textes des $16^{\text {ème }}$ et $18^{\text {ème }}$ siècles, l'usage (iv) en est très proche. Il se pourrait, d'ailleurs, qu'il en soit issu.

13. Bien qu'il ait été adopté dans de nombreux travaux sur le verbe basque (entre autres, Lafon 1943, Lakarra 1996), le terme « aoriste » peut porter à confusion dans la mesure où il n'a pas la même définition dans toutes les descriptions de langues (cf. Comrie 1976: 12). Nous l'avons remplacé par un terme plus neutre, celui de « passé narratif » (Creissels 2006).

14. Emploi déjà distingué par Lafon : "Entre eguin içan da et eguin içan cen il y a la même différence qu'entre eguin da et eguin cen : les formes surcomposées du second groupe s'opposent à celles du premier comme les formes simples et les formes simplement composées s'opposent d'un groupe à l'autre. Toutefois, Liçarrague se sert parfois du parfait surcomposé, forme du premier groupe, pour rendre le passé simple du français, qu'il rend régulièrement par des formes composées ou surcomposées du second groupe. » (Lafon 1943-II : 120)

15. «Elles expriment le résultat d’une action antérieure indéterminée, généralement située dans un passé lointain : ex. qu'èi avut cantat = j'ai (eu) chanté > il m'est arrivé de chanter ; qu'a avut balhat, aquesta tèrra = elle a (eu) donné cette terre > elle a été rentable dans le passé, il y a longtemps » (Birabent \& Salles-Loustau $1989: 114)$. 
causitcen baitira falac eta garba eppaitecoac, halatan laster eguin içatu dut landa eder, çabal eta aberats hetara. (ES, Hats. 284) "Quia tamen historiae et auctorum scripta sunt decori et spatiosi agri ubi spicarum fasciculi et fasces reperiuntur metendi, ideo in ea speciosa, vasta et copiosa arva cucurri. »

(4) Gaizqui ceuden guciac senda citzan : Compli ledinçát Esaias prophetáz erran içan cena, cioela, Hark gure langoreac hartu ukan ditu, eta gure eritassunac ekarri ukan ditu (Leiz. Mt. VIII, 17) « [il] guarit tous les malades : Afin que fust accompli ce qui auoit esté dit par le Prophete Isaie, disant, Il a prins nos langueurs \& a porté nos maladies. »

(5) Iaincoac manatu vkan du, dioela ... (Leiz. Mt. XV, 4) « car Dieu a commandé, disant...»

(6) Hypocritac, vngui prophetizatu vkan du çueçaz Esaiasec, dioela (Leiz. Mt. XV,7) « hypochrites, Isaie a bien prophétizé de vous, disant...»

(7) Cõciẽciaren examinã confunditcẽ diraden personac, eta ecin averiguatu debeã aec euren pecatuen numero ciertoa, edo contua ciertoa ecin ajustadu debenac cembat pecatu eguin izan debean Mandamentu bacoitzaren contra essan beze cembat tempora egon diraden pecatuan. (OA 133) 'Que les personnes qui, lors de l'examen de leur conscience se trompent, et celles qui ne peuvent délivrer le nombre exact de leurs péchés, ou ne peuvent dire de façon précise combien de péchés elles ont commis à l'encontre de chaque commandement, disent combien de temps elles sont restées dans le péché'.

(8) Orai nahi nuque mintçatu Laburdi edo Lau-urdi ene herriaz, batere mintçatu gabe haren abantaillez eta, bere merecimenduen cariez, erreguenganic ardietsi içatu tuen libertate eta garacia bereciez. (ES, Hats. 201) « Nunc pervelim de Laburdo sive de Lau-urdo patria mea loqui; nec tamen commemorabo de eius dotibus, facultatibus et praerogativis, quas pro suis meritis a regibus adepta est. »

(9) Ecen Propheta guciéc eta Legueac Ioannesganano prophetizatu vkan duté (Leiz. Mt. XI, 13) «tous les prophètes ont prophetizé jusqu'à Iean »

(10) nic behintçat, içatu naicen herri guztietan, bethi entçun içatu dut (bat bederac entçun ahal içatu duquen beçala), guztiec aho batez aithor hau eta laudamen bera emaiten diotela Saraco escuarari. (ES, Hats. 198) « Et ut modo verum palam patefaciam, ubique enim incolui semper audivi, quemadmodum unusquisque audire potuerit, omnes unanimiter hunc assensum ipsumque suffragium Sarensi Cantabrismo tribuere.»

(11) egundainotic munduco gendaqui guciec estimu handitan iduqui içatu tuztela, ceinec bere herrico hitzcuntça. (ES, GC38) 'Toutes les nations du monde ont depuis toujours eu une grande estime pour leur langue'.

(12) Guiçonen artian içan niçan ordu oroz, gutiago guiçon etcherat utçuli içan niç (Mst 52) «toutes les fois que j'ai été dans la compagnie des hommes j'en suis revenu moins homme que je n'étais»

(13) Orduan ioan içan da hamabietaric bat Iudas Iscariot deitzen cena, Sacrificadore principaletara. (Leiz. Mt. XXVI, 14-15) « Adonc l'vn des douze qui estoit appelé Iudas Iscariot, s'en alla aux principaux sacrificateurs. ».

(14) edo erranbeharda, herri hautarat ethorri izandiren jendaqui arrotcec Escuaratic harturic hitçac, eta jcenac berequin eramanizantuztela bere herrietarat (ES, Hats. 41) «Vel dicendum extraneos qui ad istam patriam pervenerant vocabula et nomina a Cantabrismo secum adduxisse ad patriam suam. »

(15) Artha bera içatu içan dute hainitz bertce emperadorec eta erregueec, eta nihor 
ere ez hambat nola Luis gure erregue handiac (ES, Hats. 246) «Eamdem sollicitudinem posuerunt plures alii imperatores et reges, et nemo tantam quantam Ludovicus magnus noster rex»

Ensuite, chez Etxeberri de Sare on relève des formes hypercomposées de présent, c'està-dire avec deux participes ajoutés au participe lexical (izatu izan). Ce type de surcomposées, extrêmement rare, se retrouve en Yiddish et dans les variétés françaises du canton de Vaud (Schaden 2009). ${ }^{16}$ Comme dans ces langues, la valeur attachée à ces formes est celle de passé lointain.

(16) segur ere, hitz suerte hec lothu ahalizanzaizcola, bertce orduz Escual herrirat ethorri izatuizandiren jende arrotcetaric (ES, Hats. 40) « illius modi vocabula ei coniuncta fuisse ab exteris gentibus, quae olim venerant in Hispaniam »

(17) Halarican ere, hainitz presunaya handi eta jaquintsun içatu içan dira eguiteco huni borthizqui jarraiqui içatu içan çaizconac (ES, GC9) 'Malgré tout, de nombreux grands personnages et savants se sont attelés à cette tâche de façon très cruelle'.

En résumé, en basque ancien, les périphrases surcomposées à auxiliaire de présent ont double fonction : (i) aspectuelle de parfait (parfait, parfait d'expérience, parfait lointain, parfait itératif), c'est-à-dire qu'elles désignent un état résultant terminé mais qui conserve une certaine pertinence au moment de l'énonciation ; (ii) temporelle de passé qui n'a aucune pertinence actuelle (passé lointain, passé narratif).

\subsection{Surcomposées à auxiliaire de passé}

En basque ancien, les périphrases surcomposées à auxiliaire de passé sont également fréquentes. Elles expriment :

(i) le passé narratif (Leizarraga, Lazarraga, Otxoa Arin, Maister). Bien souvent, elles sont entourées des autres formes de passé narratif d'usage selon l'époque concernée (18-21).

(ii) l'antériorité d'un événement du passé par rapport à un autre événement passé, soit, le plus-que-parfait. Notons que cette valeur ne leur est attachée qu'en subordonnées (relatives, temporelles) ${ }^{17}$ (Leizarraga, Etxeberri de Sare) (22-

16. Le persan et le tchèque peuvent accumuler jusqu'à trois participes (actifs) (Estaji \& Bybenik $2007: 51)$

17. Selon Lafitte (1962 : 386), en basque moderne cette valeur se retrouve également en proposition principale : irakurtu izan nuen « j'avais antérieurement lu, il m'était arrivé de lire ». 
(iii) le passé lointain (Otxoa Arin, Etxeberri de Sare, Maister, Beriain) (26-29).

(18) Eta ilkiric Iesusec ikus ceçan gendetze handibat eta compassione har ceçan heçaz, eta hayén arteco eriac senda citzan. Eta arrats aldean ethorri içan çaizcan bere discipuluac (Leiz. Mat. XIV, 14-15) «Et Iesus sortant vid une grande multitude, et eut compassion d'eux, et guarit les malades d'entr'eux. Or comme le soir venoit, ses disciples vindrent à luy »

(19) Egun batean leucala / beste baçugaz cabildu, / aen artera içan ninçan / exilexilic coladu (Laz B12: 47-48) 'un jour, alors qu'elle était réunie avec d'autres, je m’introduisis discrètement parmi eux

(20) bear ditu penitenteac clarecireaquin [...] essan bere culpac, eta pecatu guziac, cembat eta cer moetacoac eguin izan cituan (OA 129) 'Le pénitent doit dire toutes ses fautes et péchés avec clarté, combien et de quels types [de péchés] il commit'

(21) Guiçonbat behin [...] ceri egon leitian ecin haitatuç, ardura çagouelaric eskerniaturic [...] aldi bateç eliçabatetan [...] oracionetan jarri içan cen (Mst. I, ch. XXV, 2) «Un homme qui flottait souvent, plein d'anxiété, [...] un jour [...] entra dans une église; et, se prosternant devant un autel pour prier...»

(22) Han ciraden halaber anhitz emazte urrundanic beha ceudela, cein iarreiqui içan baitzaizcan Iesusi Galileatic (Leiz. Mt. XXVII, 55) « Il y avoit là aussi plusieurs femmes regardans de loin, lesquelles auoyent suivi Jesus de Galilee »

(23) Informa cedin diligentqui, içarra aguertu içan çayen demboráz (Leiz. Mt. II, 7) « [il] s'enquit d'eux songneusement du temps que l'estoille leur estoit apparue »

(24) Eta etchean sarthu içan cenean, aitzin cequión Iesus, cioela, Simon, cer irudi çaic ? (Leiz. Mat. XVII, 25) «Et quand il fut entré en la maison, Iesus s'auança, et luy dit, Simon, que t'en semble?»

(25) asco lekhutan eguin içan çuten beçala (ES, Hats. 61) « ut pote in pluribus locis executi fuerant $»$

(26) Momentu onetan, bada, Verbo Eternoa, leen solamente Jaungoicoa zana [...] asi izandu zan [...] guizon izaten (OA 27) 'Donc, à ce moment là, le Verbe Eternel, qui jusqu'alors était seulement Dieu [...] commença à être homme'

18. La forme composée simple est également employée dans ce contexte. Comparer (24) ci-dessus et l'exemple suivant: Eta sarthu cenean Iesus Capernaumen, ethor cedin harengana Centenerbat othoitz eguiten ceraucala (Leiz. Mat. VIII, 5) « quand il fut entré ». Par ailleurs, en subordonnées la forme surcomposée ne véhicule pas toujours un sens d'antériorité : Eta ençun ukan çuenean Iesusec, ecen Ioannes presóner cela, retira cedin Galileara (Leiz. Mat, IV, 12) « Or quand Iesus entendit que Iean estoit prisonnier, il se retira en Galilee »; Hunqui vkan çuten guciac senda citecen (Leiz. Mat. XIII, 36) «tous ceux qui le touchèrent furent guaris »

19 «Les formes surcomposées reliées à un temps composé ont pour fonction d'établir une chronologie : elles situent une action antérieurement à celle du temps composé » ex. Talèu aui èi avut escriut, que soi sortit « aussitôt que j'ai eu écrit, je suis sorti » (Birabent \& Salles-Loustau 1989 : 114). 
(27) Julio Cesari bere etsajac hiltcera joan citçaizconean, eta hil içan baitçuten hogoy eta hirur traqueta colpez (ES, Hats. 214-15) « quando ad Iulium Caesarem interficiendi gratia eius inimici pervenerunt, cuique mortem viginti et tribus pugionis ictibus attulerunt»

(28) Erromaren contra jaiqui içan cen Hetruria (ES, GC 38) 'L'Etrurie se souleva contre Rome'

(29) Davit, erregue ecinago debota bere indar oroç, Jincouaren arkharen aitcinian dantçatu içan cen, orhitcen celaric Jincouac besteorduç haren aiter eguin çutian hountarçuneç (Mst. 336)

Autrement dit, les périphrases surcomposées à auxiliaire de passé revêtent deux valeurs principales : (i) l'antériorité (plus-que-parfait, passé lointain) ; (ii) le passé narratif.

\section{Evolution diachronique}

\subsection{Calques des langues romanes?}

Selon Lafon (1943, II-120), les formes surcomposées du basque « ont sans doute été créées à l'imitation de celles du français », et, comme nous l'avons vu plus haut (4me note de bas de page), il pense qu'en basque ancien leur usage était limité à l'écrit, et plus particulièrement à celui de Leizarraga.

Grâce aux données des $17^{\text {ème }}$ et $18^{\text {ème }}$ siècles, nous pouvons apprécier que la majorité des emplois des surcomposées ne sont pas spécifiques à la traduction de Leizarraga. D'ailleurs, chez ce dernier auteur, elles ne semblent pas non plus être calquées sur le texte source car il suffit de comparer la version française du Nouveau Testament de $1563^{20}$ pour remarquer que les emplois surcomposés de Leizarraga ne correspondent pas à des emplois surcomposés du français :

(30) Eta ereitean hacitic batzu eror citecen bide bazterrera : eta choriac ethorri içan dirade, eta iretsi vkan dituzte hec. Eta batzu erori içan dirade leku harriçuetara, non ezpaitzuten heuragui lurric [...] Guero iguzquia goratu eta, erre içan dirade, eta ceren ezpaitzuten erroric, eyarthu içan dirade. Eta batzu erori içan dirade elhorri artera : eta handitu içan dirade elhorriac, eta itho vkan dituzte hec. (Leiz, Mt, XIII, 4-7) « Et comme il semoit, vne partie de la semence est cheuute aupres du chemin, et les oiseaux sont venus et l'ont deuoree. Et l'autre est cheute en lieux pierreux, ou elle n'auoit guere de terre. [...] Et le solei estant leué, elle a esté hallee: et pource qu'elle n'auoit nulle racine, elle est sechee. Et l'autre est cheute entre les espines: et les espines sont montees, et l'ont estouffee. »

Quant à l'influence des langues environnantes, les données chronologiques sont intéressantes. Ces formes apparaissent en français (Ayres-Bennett \& Carruthers 1992) et en occitan (Ronjat [1930-41] 1980) dès le début du $16^{\text {ème }}$ siècle. Néamoins, les fonctions des

20. Il semblerait que ce soit la version la plus proche de la traduction de Leizarraga (Salaberri Muñoa 2015). 
périphrases surcomposées dans ces langues et en basque ne sont pas toutes les mêmes. En français, elles ont deux emplois principaux : (i) en subordonnées elles expriment l'antériorité, le verbe principal étant une forme composée simple (quand j'ai eu lu mon livre, je suis sorti ; quand il a eu fini de déjeuner, il s'est endormi); (ii) en proposition principale, la perfectivité, et dans quelques cas, la relative rareté de l'événement : j'ai eu fait les foins à la main ; il a eu coupé, ce couteau (Ayres-Bennett \& Carruthers 1992). En occitan, elles expriment : (i) le parfait lointain (« superparfait »), c'est-à-dire la permanence du résultat d'un passé lointain (cf. $11^{\text {ème }}$ note de bas de page); (ii) l'antériorité en subordonnées : quand aguèt agut ausit aquò, sortiguèt de l'ostal 'quand elle eut entendu cela, elle quitta la maison' (Wheeler 1988). Par ailleurs, on retrouve ces formes dans les dialectes péninsulaires dont la langue de contact (l'espagnol) n'a pas développé de formes de ce type.

Nous verrons ci-dessous que les périphrases surcomposées ont plus vraisemblablement été créées par et pour le système verbal basque, afin d'apporter des nuances aspectuelles et temporelles nouvelles. Il n'empêche que des phénomènes d'aire linguistique (sprachbund) doivent, selon nous, tout de même envisagés.

\subsection{De la nécessité des périphrases surcomposées}

On peut également se poser la question de la nécessité des périphrases surcomposées dans le système verbal ancien. En effet, au $16^{\text {ème }}$, comme aux $17^{\text {ème }}$ et $18^{\text {ème }}$ siècles, la langue basque possède d'autres formes pour exprimer le parfait, le parfait d'expérience, le passé narratif et le plus-que-parfait. En revanche, il semblerait qu'elle n'apporte les valeurs de parfait lointain, parfait itératif et passé lointain qu'à l'aide de ces constructions surcomposées.

Dès le $16^{\text {ème }}$ siècle, la langue basque dispose de la périphrase composée du participe passé et des auxiliaires izan « être » et *edun « avoir » de présent pour exprimer le parfait (3133). C'est la forme de parfait la plus courante en basque ancien comme de nos jours. Létude l'évangile de St Mathieu de Leizarraga et des textes de Maizter, Etxeberri de Sare et Otxoa Arin ne revèle aucune différence sémantique entre cette périphrase et la forme surcomposée ; elles semblent absolument synonymes et interchangeables (37). Elles sont en étroite compétition chez Leizarraga: dans ledit texte, la forme surcomposée représente un peu plus d'un tiers des occurrences des formes de parfait (58 occurrences contre 101 occurrences de la périphrase [participe passé + izan « être » / edun « avoir »]). Ces données sont d'autant plus surprenantes que les formes surcomposées à auxiliaire de présent ne sont pas employées dans les autres textes de l'époque. Au $18^{\text {ème }}$ siècle, elle est vraiment marginale dans ce contexte (3436): chez Maizter, on la relève 4 fois contre 101 fois la périphrase [participe passé + izan « être » / *edun « avoir »], chez Etxeberri de Sare et Otxoa Arin, les proportions sont les suivantes : 2 vs 249 et 2 vs 198, respectivement. Enfin, la périphrase [participe passé + izan « être » / *edun «avoir »] peut aussi prendre valeur de parfait d'expérience (38-40). Néanmoins, elle ne revêt jamais les valeurs de « parfait lointain » et de «parfait itératif ».

(31) Eztuçue iracurri Dauid-ec gossez eguin çuena (Leiz. Mt. XII, 3) « N'auez-vous point leu ce que fit Dauid ayant faim ?»

(32) ayta-amaoc efini dave / Flora oy encerraduric (Laz. Al1 : 23-24) « les parents ont enfermé Flora »

(33) Adausia eta ausikia behinkoaz ukhen tut (Oih. Prov. 1) « J'ai été aboyé et mordu à la fois » 
(34) Modu onetan oracioan exercitaduric, bear ditu Christaubac dilligencia, eta cuidadu prudente batequin examinatu bere vici gucico temporan eguin dituan pecatuac (OA 132) 'Ainsi exercé dans la prière, le Chrétien doit examiner avec une application et une attention prudentes les péchés qu'il a commis tout au long de sa vie'.

(35) Khambiatu deitaçu, dio, ene plagnia alagrantciala, eta boçtarioç unguratu naiçu (Mst. II, ch.IX, 5) «Vous avez, dit-il, changé mes gémissements en chants d'allégresse, et vous m'avez environné de joie »

(36) Ecusi det nola daquizun cer sinistu bear dezun (Iraz. 21) 'J'ai vu que tu sais qu'est-ce que tu dois croire'

(37) Arrotz nincen, eta recebitu nauçue, billuci, eta veztitu nauçue : eri, eta visitatu nauçue : Presoindeguian nincén, eta enegana ethorri içan çarete (Leiz. Mt. XXV, 3536). « i'estoye estranger et vous m'auez recueilli, i'estoye nud, et vous m'auez vestu, i'estoye malade et vous m'auez visité, i'estoye en prison et vous estes venus à moy »

(38) Egundano ezta aguertu hunelaco gauçaric Israelen (Leiz. Mt. IX, 33) « Onques ne fut veue chose semblable en Israel »

(39) Secula eçtut ihour ediren, çoumbat nahi beita religious, eta debot cen, bere graciaren gabeciac çoumbait aldiç ukhen eçtutianic (Mst. II., ch. IX, 7) « Je n'ai jamais rencontré d'homme si pieux et si parfait qui n'ait éprouvé quelquefois cette privation de la grâce.»

(40) dela egundaino nihorc aditu othe du ukhoric eguin dioela fede saindu huni? (ES, Hats. 105) « sive aliquis unquam audivitne eam destituisse hanc sanctam fidem »

Le passé narratif jouit de différentes formes. Au $16^{\text {ème }}$ siècle, il peut être exprimé par (i) les formes synthétiques de passé (41-42), (ii) la périphrase composée du radical verbal ou du participe passé et des auxiliaires *edin « devenir », *ezan et egin « faire » de passé (43-44), (iii) la périphrase composée du participe passé et des auxiliaires izan « être » et *edun « avoir » de passé (45-46). Leizarraga et Lazarraga emploient de surcroit les périphrases surcomposées, le premier celles à auxiliaire de présent et de passé, le second seulement celle à auxiliaire de passé. Néanmoins, ses apparitions sont très sporadiques : chez Leizarraga dans l'évangile de Mathieu, on les compte 9 fois vs 609 fois la périphrase (ii) et 2 fois la périphrase (iii); chez Lazarraga, une seule fois contre 158 fois la périphrase (iii). De même, au $18^{\text {ème }}$ siècle, alors que la périphrase [participe passé + izan / *edun de passé] est devenue la forme de passé narratif la plus courante dans toutes les variétés dialectales, la périphrase surcomposée demeure très rare.

(41) Jagui çan bere sillarean, / nic au esan da bertati ; / artu ninduan escurean, / baguioancen arerean / floresta baten erditi (Laz. B18 : 81-85) «Elle se leva de sa chaise, / sitôt ai-je dit cela; / elle me prit par la main, / nous partîmes de là / à travers un bosquet »

(42) Gure mandoa urac engarren eta urac aroa (Gar. 139-19) « Nuesto macho el agua te traxo, y el agua te lleva »

(43) Ioan nendin enaguien oguen gabe ihesic (Etxp. XIII, 10) « J'y allai : étant innocent, je ne pris point la fuite»

(44) Etxea urra zezana egur egiteko, xaz bero zedin, aurten hotzez hiltzeko (Oih. Prov. 517) «Celui qui défit sa maison pour en tirer du bois à chauffer se chauffa l'année passée, pour mourir de froid cette année »

(45) Saynduyac eçiraden sarthu vanitatez glorian (Etxp. I, 167) « Les saints n'entrèrent pas dans la gloire par vanité » 
(46) Bi hautaric ceinec eguin çuen bere aitaren vorondatea? (Leiz. Mat. XXI, 31) « Lequel de ces deux fit la volonté du père?»

Le plus-que-parfait, lui, est généralement exprimé par la périphrase composée du participe passé et des auxiliaires izan « être » et *edun « avoir» de passé. La périphrase surcomposée de passé est plus rarement employée que celle-ci.

(47) [...] advertidu beza [...] ote ciraden voto castidadecoa eguin ceban personàc (OA 159) 'qu'il dise s'il s'agissait de personnes qui avaient fait voeux de chasteté'.

(48) Gudu edo estrosada hunetan, gortheco presunaja guehienac, erreguec harmadaco aguintari eguin cituenac, hillac guelditu ciren, eta bagayeriac galduac. " In hoc certamine plerique aulicorum, quos rex copiis praefecerat, interfecti sunt, direpta impedimenta, $<81>$ et hostis propter notitiam locorum statim in diversa dilapsus est. » (ES, Hats. 80-81)

Néanmoins, dans le corpus étudié aucune de ces formes n'exprime le passé lointain ; dans ce cas, les auteurs ont recours aux formes surcomposées.

\subsection{Création et évolution des périphrases surcomposées}

\subsubsection{Surcomposées à auxiliaire de présent}

On l'a dit, dès les premiers textes, on relève une autre forme de parfait, la périphrase [participe passé + izan « être »/*edun « avoir »]. Au 16 $6^{\text {ème }}$ siècle, cette construction prend également valeur de résultatif ${ }^{21}$ (49-53) dans les textes labourdins (Leizarraga), bas-navarrais (Etxepare) et souletins (Oihenart, Zalgize). Tout semble indiquer qu'il s'agit à l'origine d'une périphrase de résultatif par la suite réanalysée en parfait (Mounole 2011).

(49) Eguin denean, gehennaco seme eguiten duçue dobláz (Leiz. Mt. XXIII, 15) « quand il est faict, vous le rendez fils de gehenne au double plus que vous »

(50) Ene cecenac eta haraquey guicenduac haraqueitatu dirade (Leiz. Mt. XXII, 4) « mes taureaux et mes bêtes engraissées sont tuées »

(51) çamariac joan direnian, establia cerra (Zalg. 65) « Lorsque les chevaux de selle sont partis, ferme l'étable »

(52) Habia egin deneko, xoria hil (Oih. Prov. 206) « Pour lors que la cage a été faite, l'oiseau est venu à mourir »

(53) Haur merda, gurentu denean alfer da (Oih. Prov. 220) « Un enfant nourri trop délicatement est fainéant quand il est devenu grand »

Il se pourrait que la périphrase surcomposée soit survenue pour éviter les ambiguités aspectuelles véhiculées par la périphrase [participe passé + izan « être » / *edun « avoir »], l'ajout du participe « extra » permettant de renvoyer l'action passée et son résultat dans la

21. Le résultatif exprime un état qui est donné comme le résultat d'une action passé (Comrie 1976, Bybee \& al. 1994). 
sphère du passé pour n'en conserver qu'une éventualité au moment de l'énonciation. D'ailleurs, exception faite de Otxoa Arin, c'est dans les parlers où la périphrase [participe passé + izan « être » / *edun « avoir »] a conservé sa double valeur le plus longtemps que s'emploie la périphrase surcomposée à auxiliaire de présent pour faire référence au parfait (cf. Leizarraga, Otxoa Arin, Maizter).

Les périphrases surcomposées du basque, comme celles du français et du danois, n'ont jamais valeur résultative (Schaden 2009); au contraire, dans ces trois langues, on y a recours pour éviter cette interprétation. ${ }^{22}$ Ces données semblent donc indiquer que dans certaines langues les périphrases surcomposées apparaissent en tant que formes de parfait. Ceci va à l'encontre l'hypothèse de Bybee \& al (1994) selon laquelle tout parfait est issu du résultatif, et fragilise la proposition de Aldai (2002) concernant l'évolution diachronique des surcomposées du basque.

La périphrase surcomposée à auxiliaire de présent du basque, apparue comme forme de parfait, a pu rapidement exprimer le parfait d'expérience : si les deux désignent une situation du passé dont les conséquences sont toujours pertinentes au moment de l'énonciation, la seconde spécifie que cette situation s'est produite au moins une fois dans le passé. Le parfait itératif, lui, s'explique à partir du parfait d'expérience : on passe d'une d'une situation accomplie au moins une fois, à sa répétition. En ce qui concerne le parfait lointain (superparfait), il semble découler de ce participe qui émet une nuance d'antériorité. Ensuite, c'est de cette notion antériorité que sont issus le passé lointain, puis le passé narratif (Squartini 1999, Schaden 2009). ${ }^{23}$

En basque moderne, la périphrase surcomposée à auxiliaire de présent exprime parfois le passé lointain, mais jamais le passé narratif. La périphrase [participe passé + izan « être » / *edun « avoir »], à auxiliaire de passé dans les dialectes péninsulaires (etorri nintzen, egin nuen), et à auxiliaire de présent dans les dialectes continentaux (etorri naiz, egin dut), s'étant généralisée, l’usage de la forme surcomposée dans ce contexte est tombé en désuétude.

\subsubsection{Surcomposées à auxiliaire de passé}

Comme décrit plus haut (\$3.2), en basque ancien comme de nos jours, la périphrase

22. « On ne peut pas non plus affirmer que les temps résultatifs sont une tentative pour regagner une valeur résultative perdue dans un processus de grammaticalisation. Les premières données du français et les données du danois semblent plutôt pointer dans la direction inverse : les surcomposés sont, à l'origine, une tentative pour éviter un inférence avec un état résultant. » (Schaden $2009: 251$ ).

23. «Résumons donc : des emplois de type superparfaits existent également dans les variétés méridionales de l'allemand, mais les contextes d'utilisation ne sont pas toujours identiques entre l'allemand et le français, ni même à l'intérieur des variétés allemandes. Le fait qu'il existe des langues comme le danois ou le breton, dans lesquelles il existe uniquement des superparfaits, et pas de surcomposés antérieurs, tend à conforter une vue selon laquelle (i) les superparfaits sont la source diachronique des temps surcomposés; et (ii) les superparfaits se distinguent, au moins dans certaines langues, ou à certaines étapes du développement d'une langue, dans leur sémantique des surcomposés antérieurs. » (Schaden 2009 : 215). 
composée du participe passé et des auxiliaires izan « être » et *edun « avoir » (etorri nintzen, egin nuen) exprime le plus-que-parfait et le passé narratif. C'est du contexte que se dégage l'une ou l'autre valeur aspectuelle. Au $16^{\text {ème }}$ siècle, elle revêt en plus la valeur résultative de passé chez Leizarraga (54-57). A l'instar de la périphrase à auxiliaire de présent, il semblerait que cette construction était initialement une forme de résultatif passé et qu'elle se soit par la suite grammaticalisée en plus-que-parfait, puis en passé narratif (Mounole 2011).

(54) Hura bera, harequin crucificatu ciraden gaichtaguinec-ere reprochatzen ceraucaten (Leiz. Mt. XXVII, 44) «Cela mesme luy reprochoyent aussi les brigans qui estoyent crucifiez auec luy ».

(55) Eta harrapatzen du haren bihotzean erein cena (Leiz. Mt. XIII, 19) « [Il] rauit ce qui est semé au cœur »

(56) Ecen ethorri içan da guiçonaren Semea galdu cenaren saluatzera (Leiz. Mt. XVIII, 11) «car le fils de l'homme est venu pour sauver ce qui était péri »

(57) Çacussanean hura condemnatu cela (Leiz. Mt. XXVII, 3) «Voyant qu'il estoit condamné »

La périphrase surcomposée à auxiliaire de passé, comme celle à auxiliaire de présent, n'a jamais valeur résultative dans les textes les plus anciens. Comme en français (Schaden 2009: 243), il semblerait même que Leizarraga y ait recours pour éviter l'interprétation résultative que la périphrase simple aurait véhiculée, et attacher au verbe la valeur d'antériorité propre au plus-que-parfait. C'est de cette nuance d'antériorité qu'est issu le passé lointain comme en témoigne l'évolution du plus-que-parfait de nombreuses langues telles que le latin, l'hindu-ourdou, l'arménien, le bengali et l'amharique (Comrie 1985, Squartini 1999). ${ }^{24}$ Le passé lointain peut par la suite perdre cette distance par rapport au moment de l'énonciation indiquer un passé plus général et de là exprimer le passé narratif (Squartini 1999).

L'émergence des formes surcomposées est fréquemment expliquée par le déplacement de la périphrase de parfait vers des emplois de passé narratif. ${ }^{25}$ C'est à première vue une explication convaincante, dans la mesure où le français, les langues rhéto-romanes, les dialectes septentrionaux de l'italien, l'allemand et le yiddish qui possèdent des périphrases surcomposées, ont tous vu leur forme de parfait développer l'usage temporel de passé narratif au détriment des formes de passé simple qui sont éliminées progressivement (Schaden 2009 : 202). Cependant, en français, l'émergence des surcomposées concerne d'abord le plus-que-

24. "The change of past anterior to remote past is a generalization of meaning and parallels the change of anterior to simple past. Since in a past narrative the past anterior is used for situations that are prior to those signaled in the simple past, past anterior situations are more remote than past ones, and at the same time, relevant in some way to the past situation. » (Bybee \& al.1999: 102).

25. «Une des explications standards de la genèse des temps surcomposés du français, avancée parmi d'autres par Foulet (1925), est que les formes surcomposées constituent une tentative pour regagner une forme aspectuellement résultative, au moment où le passé composé devient de plus en plus prétérital, et perd progressivement sa valeur originelle de résultatif. » (Schaden 2009 : 199). 
parfait, ensuite les emplois superparfaits précèdent les emplois antérieurs, qui eux peuvent être liés au renouvellement du passé narratif. ${ }^{26}$ De plus, elles apparaissent dans des langues où le passé simple est encore vivant (occitan, dialecte français du Morvan) (Schaden 2009).

En basque, cette explication ne tient pas non plus. Certes, en basque aussi la périphrase [participe passé + izan / *edun] à auxiliaire de présent a déplacé la forme de passé narratif [participe passé + izan / *edun] à auxiliaire de passé. Cependant, cela ne s'est produit que dans les dialectes continentaux et ce, courant $18^{\text {ème }}$ siècle, autrement dit, bien après l'apparition des périphrases surcomposées.

De nos jours, la périphrase surcomposée à auxiliaire de passé a perdu cette fonction de passé narratif. Les périphrases simples s'étant généralisées à ce tiroir verbal (celle à auxiliaire de présent dans les dialectes septentrionaux et celle à auxiliaire de passé dans les dialectes péninsulaires), son usage a peu à peu disparu.

\section{Conclusion}

En basque, les périphrases surcomposées apparaissent dès les premiers textes. Bien qu'elles occupent une place plutôt marginale dans le système verbal, elles ne sont pas négligeables dans la mesure où elles apportent de nouvelles nuances aspectuo-temporelles. Il semblerait que les surcomposées à auxiliaire de présent, comme celles de passé, aient été créées afin remédier à l'ambigüité aspectuelle des périphrases [participe + izan « être »/*edun « avoir »] de présent et de passé et apporter une interprétation de parfait sans équivoque aux énoncés. De plus, le participe « extra » leur octroie une nuance d'antériorité qui apporte à la langue la possibilité d'exprimer le parfait lointain et le passé lointain. Les évolutions postérieures qui ont fait d'elles de potentielles formes de passé narratif n'ont pas abouti, à ce tiroir verbal, les périphrases [participe + izan « être »/*edun « avoir»] de présent et de passé s'étant généralisées, dans les dialectes continentaux et de passé dans les dialectes péninsulaires respectivement.

\section{Références}

Aldai, Gontzal. 2002. Grammaticalization of present and past in Basque. Thèse doctorale: Universtity of California.

26. « Diachroniquement, les formes surcomposées attestées en français concernent donc d'abord le plus-que-parfait, temps qui ne devrait pas être inquiété par la dérive prétéritale du passé composé. De plus, si les surcomposés antérieurs sont bien liés à cette dérive prétéritale, on est obligé de constater que les emplois des surcomposés en tant que superparfaits sont plus anciennement attestés que les surcomposés antérieurs. » (Schaden 2009: 247). "Pour le français, on voit clairement que l'apparition des temps surcomposés n'est pas liée à la disparition du passé simple. Tout au plus, on pourrait affirmer que l'affaiblissement du passé simple, et avec lui, du passé antérieur, a entraîné une augmentation de la fréquence d'utilisation du passé surcomposé. » (Schaden 2009 : 251). 
Aldai, Gontzal. 2008. From ergative case marking to semantic case marking: the case of historical Basque. In Mark Donohue \& Søren Wichmann (éds.), The typology of semantic alignment, 197-218. Oxford: Oxford University Press.

Ayres-Bennett, Wendy \& Carruthers, Janice. 1992. Une regrettable et fort disgracieuse faute de français? The description and analysis of the French surcomposés from 1530 to the present day. Transactions of the Philological Society 90. 219-257.

Birabent Jean-Pierre \& Salles-Loustau Jean. 1989. Memento grammatical du gascon. Pau : Escòla Gaston Febus \& Nosauts de Bigòrra.

Bybee, Joan, Perkins Revere \& Pagliuca, William. 1994. The evolution of grammar: Tense, aspect and modality in the languages of the world. Chicago / Londres: The University of Chicago Press.

Comrie, Bernard. 1976. Aspect. Cambrigde : Cambridge University Press.

Comrie, Bernard. [1985] 1993. Tense. Cambrigde : Cambridge University Press.

Creissels, Denis. 2006. Syntaxe générale, une introduction typologique. Paris : Hermès.

Creissels, Denis \& Mounole, Céline, 2015. Non canonical valency patterns in Basque : variation and evolution. Manuscrit.

Estaji Azam \& Bubenik Vit. 2007. On the development of the tense / aspect system in Early New and New Persian. Diachronica XXIV-1. 31-55.

Euskaltzaindia. [1987] 1997. Euskal gramatika, Lehen urratsak II. Bilbao : Euskaltzaindia.

Harris. Martin. 1982. The 'past simple' and the 'present perfect' in Romance. In Nigel Vincent \& Martin Harris (éds), Studies in the Romance Verb, 42-70. Londres : Croom Helm.

Harris. Martin. 1988. French. In Martin Harris \& Nigel Vincent (éds), The Romance Languages, 209-245. Londres : Routledge.

Lakarra. Joseba Andoni. 1996. Refranes y Sentencias (1596): Ikerketa eta edizioa. Euskaltzaindia: Bilbao.

Lafon. René. 1943. Le système du verbe basque au XVIème siècle. Bordeaux : Delmas.

Mounole. Céline. 2011. Le verbe basque ancien : étude philologique et diachronique. Thèse de doctorat. UPV/EHU \& Université Bordeaux III Michel de Montaigne.

Mounole. Céline. 2014. Lazarragaren gramatika. ASJU 48. En cours d'impression.

Oyharçabal. Bernard. 1987. Etude descriptive de constructions complexes en basque: Propositions relatives, temporelles, conditionnelles et concessives. Thèse de doctorat d'Etat ès Lettres et Sciences Humaines. Paris : CNRS.

Oyharçabal. Bernard. 2003. Tense, aspect and mood. In José Ignacio Hualde \& Jon Ortiz de Urbina (éds), A Grammar of Basque, 249-284. Berlin/New York: Mouton de Gruyter.

Ronjat. Jules. [1930-41] 1980. Grammaire istorique des parlers provençaux modernes (4 vols). Genève-Marseille: Stlakine-Laffitte.

Salaberri Muñoa. Patxi. 2015. Leizarragaren idazlanetan barrena. Bilbao : EHU/UPV.

Shaden. Gerhard. 2009. Composés et surcomposés : le 'parfait' en français, allemand, anglais, espagnol. Paris : LHarmattan.

Squartini. Martin. 1999. On the semantics of the Pluperfect: Evidence from Germanic and Romance. Linguistic Typology 3. 51-89.

Wheeler. Max. 1988. Occitan. In Martin Harris \& Nigel Vincent (éds), The Romance Languages, 246-278. Londres : Routledge. 